\title{
Safety and effectiveness of daily teriparatide for osteoporosis in patients with severe stages of chronic kidney disease: post hoc analysis of a postmarketing observational study
}

\author{
This article was published in the following Dove Press journal: \\ Clinical Interventions in Aging \\ 15 November 2016 \\ Number of times this article has been viewed
}

\author{
Atsushi Nishikawa' \\ Fumito Yoshiki \\ Masanori Taketsuna ${ }^{2}$ \\ Kenta Kajimoto ${ }^{2}$ \\ Hiroyuki Enomoto 2 \\ 'Global Patient Safety Japan, Quality \\ and Patient Safety, Eli Lilly Japan K.K., \\ ${ }^{2}$ Medicines Development Unit Japan, \\ Eli Lilly Japan K.K., Kobe, Japan
}

\begin{abstract}
Teriparatide (recombinant 1-34 N-terminal sequence of human parathyroid hormone) for the treatment of osteoporosis should be prescribed with caution in patients with severe stages of chronic kidney disease (CKD). However, in clinical settings, physicians and surgeons who treat such patients have few available options. We sought to further explore the safety and effectiveness of teriparatide for the treatment of osteoporosis in Japanese patients with severe stages of CKD. This was a post hoc analysis of a postmarketing surveillance study that included patients with osteoporosis at high risk of fracture and stage 4 or $5 \mathrm{CKD}$. Patients received subcutaneous teriparatide $20 \mu \mathrm{g}$ daily for up to 24 months. Safety profiles were assessed by physician-reported adverse drug reactions (ADRs). Effectiveness was assessed by measuring bone formation (via procollagen type $1 \mathrm{~N}$-terminal propeptide [P1NP]), bone mineral density (BMD), and the incidence of clinical vertebral or nonvertebral fragility fractures. A total of 33 patients with severe stages of CKD (stage 4, n=30; stage 5, n=3) were included. All patients were female, and $81.8 \%$ had a history of previous fracture. No serious ADRs were recorded; a total of 4 ADRs were recorded for 4 of 33 patients. Increases in BMD and P1NP levels were observed both overall and in most individual patients. New fractures occurred in 1 patient with stage $5 \mathrm{CKD}$, but not in patients with stage 4 CKD. In this post hoc analysis conducted in Japan, teriparatide appeared to be effective for the treatment of osteoporosis in elderly female patients with severe stages of CKD, and no new safety concerns were observed.
\end{abstract}

Keywords: bone mineral density, chronic kidney disease, fragility fracture, parathyroid hormone, teriparatide, safety

\section{Introduction}

Osteoporosis is a major chronic skeletal disorder characterized by decreased bone mineral density (BMD) and bone quality that increases bone fragility and risk of fracture. ${ }^{1}$ The prevalence of osteoporosis and related fractures is high and increases dramatically with increasing age. ${ }^{2,3}$ In 2009, osteoporosis was estimated to affect up to 12.8 million people in Japan. ${ }^{3}$ In addition to causing substantial morbidity and mortality from fragility fractures, osteoporosis is associated with high medical costs, which mainly relate to prolonged hospitalization and subsequent nursing care, particularly following hip fracture. ${ }^{4,5}$

Chronic kidney disease (CKD) is another common disorder that increases in prevalence with age. In $2005, \sim 13 \%$ of the Japanese adult population were estimated to have CKD and this figure is likely to be even higher in the elderly. ${ }^{6,7}$ Osteoporosis
Medical Sciences, Medicines Development Unit Japan, Eli Lilly Japan K.K.,

4-I5-I-I3F, Akasaka, Minoto-ku, Tokyo, 107-0052, Japan

Tel +81355749143

Fax +8I 355749979

Email enomoto_hiroyuki@lilly.com $\mathrm{BY}$
hereby accept the Terms. Non-commercial uses of the work are permitted without any further permission from Dove Medical Press Limited, provided the work is properly attributed. For permission for commercial use of this work, please see paragraphs 4.2 and 5 of our Terms (https://www.dovepress.com/terms.php). 
and early-stage CKD frequently coexist and $\sim 20 \%$ of patients with osteoporosis have stage 3 or $4 \mathrm{CKD} .{ }^{8}$ Further, osteoporosis and CKD are among the most common age-related comorbidities that independently impair bone strength. ${ }^{9-11}$ Early-to-moderate stages of CKD independently increase the risk of vertebral and especially nonvertebral fractures. This risk increases with worsening renal impairment., ${ }^{911-13}$

In the real-world clinical setting, there is a dilemma about how to reduce the risk of osteoporotic fracture in patients with CKD because the efficacy and safety of standard osteoporosis treatments have not been validated in these patients. Bisphosphonates (eg, alendronate and risedronate) are the main class of antiresorptive drugs and, in Japan, are considered a first-line treatment strategy for osteoporosis. ${ }^{4}$ However, according to Japanese guidelines for prevention and treatment of osteoporosis published in 2015, alendronate or risedronate should not be prescribed in patients with severe kidney disease. ${ }^{14}$

Teriparatide $\left(\right.$ Forteo $^{\circledR}$; Eli Lilly and Company, Indianapolis, IN, USA; recombinant 1-34 N-terminal sequence of human parathyroid hormone $[\mathrm{PTH}]$ ) is the first and only anabolic agent approved to treat patients with osteoporosis at high risk of fracture. ${ }^{15}$ Teriparatide regulates both bone formation and resorption, whereby intermittent exposure results in a restoration of bone microarchitecture through an increase in the number and thickness of trabeculae and accelerated mineralization. ${ }^{16,17}$ Teriparatide has been demonstrated to prevent vertebral and nonvertebral fragility fractures and to improve health-related quality of life. ${ }^{17-20}$ Careful administration of teriparatide is suggested in patients with severe renal impairment due to an observed delay in elimination in these patients compared with healthy volunteers. ${ }^{21}$ In addition, PTH is secondarily upregulated in severe stages of CKD, which potentially reduces the response to teriparatide. ${ }^{15}$ One doubleblind trial of 1,637 ambulatory postmenopausal women treated with teriparatide found that reductions in the risk of vertebral and nonvertebral fractures, as well as treatment-emergent and renal-related adverse events, were similar among patients with mild to moderate renal impairment and those without renal impairment. ${ }^{22}$ However, there is insufficient real-world evidence for clinicians to properly assess the safety and effectiveness of teriparatide treatment in patients with osteoporosis and concomitant severe stages of CKD.

A 24-month postmarketing surveillance (PMS) study demonstrated the safety and effectiveness of daily teriparatide in 1,847 patients with osteoporosis at high risk of fracture in Japan. ${ }^{8}$ Most (92.6\%) patients included in the original PMS study were female and postmenopausal (90.8\%), with a mean age of 75.4 years. ${ }^{8}$ Further, in the original PMS study, most patients were categorized as having stage $2(38.4 \%)$ or stage 3 (18.9\%) CKD, followed by stage 1 (10.5\%) CKD. Patients with severe stages of CKD (stage 4 [1.6\%], stage 5 $[0.2 \%]$ ) made up a small percentage of the study population. ${ }^{8}$ Treatment with teriparatide led to significant increases in biomarkers for bone formation/resorption and BMD, together with low incidences of new vertebral and nonvertebral fractures and significant improvements in patient-reported measurements for health-related quality of life. ${ }^{8}$ We conducted a post hoc analysis of this PMS study to explore the safety and effectiveness of daily teriparatide used in a clinical setting only among patients with severe stages of CKD and osteoporosis with a high risk of fracture.

\section{Materials and methods Study design}

This was a post hoc analysis of a prospective, multicenter, 24-month PMS study that registered 1,882 patients at 238 sites across Japan and was conducted between October 29, 2010, and February 28, 2014. ${ }^{8}$ The original PMS study was required by the Japan Ministry of Health, Labour, and Welfare and conducted in accordance with Good Postmarketing Study Practice of the Pharmaceutical Affairs Law of Japan. For this type of study, formal consent and institutional ethics approval were not required by our institutional review board.

\section{Study population}

This post hoc analysis included patients with osteoporosis at high risk of fracture and with stage 4 or $5 \mathrm{CKD}$. Specifically, osteoporosis was diagnosed using the Osteoporosis Diagnostic Criteria Review Committee of the Japanese Society for Bone and Mineral Research, 2000 revision. ${ }^{23}$ High risk of fracture was defined as low BMD, previous fracture, aging, and family history of femoral neck fracture. ${ }^{21}$ The Kidney Disease Outcomes Quality Initiative classification was used to define CKD stage. According to this classification, stage $4 \mathrm{CKD}$ is defined as an estimated glomerular filtration rate (eGFR) of 15-29 mL/min per $1.73 \mathrm{~m}^{2}$ (body surface area), whereas stage $5 \mathrm{CKD}$ (also known as end-stage renal failure) is defined as an eGFR $<15 \mathrm{~mL} / \mathrm{min}$ per $1.73 \mathrm{~m}^{2}{ }^{24}$ In this analysis, eGFR was estimated using the Modified Isotope Dilution Mass Spectrometry-Modification of Diet in Renal Disease Study equation, which has been validated to be more accurate for the Japanese population than previously reported equations. ${ }^{25}$ Exclusion criteria in the PMS study were previous teriparatide use or contraindications to 
teriparatide treatment. ${ }^{21}$ The analyses of safety, effectiveness, and fracture incidence included patients who had received at least one dose of teriparatide.

\section{Treatment protocol}

Patients received subcutaneous teriparatide $20 \mu \mathrm{g}$ daily for up to 24 months. In the PMS study, patients underwent clinical evaluations at baseline and at 1, 3, 6, 12, 18, and 24 months after the start of treatment.

However, only available measurements from all evaluations were used for this post hoc analysis. If patients discontinued treatment before 24 months, the final clinical evaluation was taken at the time of discontinuation (last visit).

\section{Safety profile}

The safety profile of teriparatide was assessed in this post hoc analysis by the same method used in the original PMS study. Adverse drug reactions (ADRs) were defined as adverse events for which a causal relation to teriparatide could not be excluded and were reported by treating physicians. ADRs were classified using preferred terms and system organ classes according to the Medical Dictionary for Regulatory Activities (Version 17.0).

\section{Effectiveness}

Effectiveness of teriparatide was assessed by measures of bone formation, bone density, and fracture incidence. Measurements of the biomarker procollagen type $1 \mathrm{~N}$-terminal propeptide (P1NP) were used to assess the effects of teriparatide treatment on bone formation. Measurements of BMD for lumbar vertebrae 2-4 (L2-L4) were used to assess the effects of teriparatide treatment on bone density according to the method preferred at each investigation center as there was no centralized laboratory. Fracture incidence was defined as the number of new clinical vertebral or nonvertebral fractures that were reported at any postbaseline visit and subsequently confirmed by radiographs.

\section{Statistical analysis}

The incidence of ADRs that occurred during the observation period was determined from physician reports. For P1NP, median values (and interquartile range [Q1, Q3]) at baseline and at 3 months, as well as the mean change $(95 \%$ confidence interval [CI]) across this interval, were calculated. For BMD, mean and standard deviation (SD) values at baseline and the last observation carried forward (LOCF), as well as the mean change across this interval (SD, 95\% CI), were calculated.
All statistical analyses were conducted using SAS Version 9.2 (SAS Institute, Cary, NC, USA).

\section{Results}

\section{Patient disposition and baseline characteristics}

Of the 1,847 patients included in the efficacy and safety analyses of the original PMS study, available data from all 33 patients with severe stages of CKD (stage 4, $n=30$; stage 5 , $\mathrm{n}=3$ ) were included in this post hoc analysis (Figure 1).

In general, baseline characteristics of this subset of patients with severe stages of CKD were similar to those of the original PMS. In terms of specific baseline characteristics, all patients with severe stages of CKD were female and most (87.9\%) were postmenopausal (Table 1$)$. The mean (SD) age was 79.8 (6.7) years and 83.0 (11.1) years in patients with stage 4 and stage 5 CKD, respectively, and most (81.8\%) patients reported a history of fracture before study registration (Table 1).

\section{Safety}

A total of 4 ADRs were recorded in 4 of 33 patients. In patients with stage $4 \mathrm{CKD}, 3 \mathrm{ADRs}$ (hyperuricemia, renal dysfunction, injection site warmth) were recorded in 3 of 30 patients. In patients with stage 5 CKD, 1 ADR (headache) was recorded in 1 of 3 patients. No serious ADRs were recorded in patients with either stage 4 or stage 5 CKD.

\section{New bone formation}

Despite the small population in this post hoc analysis, there was evidence of new bone formation among patients with severe stages of $\mathrm{CKD}$, both overall and in individual patients. Median (Q1, Q3) P1NP levels in patients with stage 4 CKD increased from $59.80 \mathrm{ng} / \mathrm{mL}(39.30,112.00 ; \mathrm{n}=9)$ at baseline to $125.55 \mathrm{ng} / \mathrm{mL}(109.00,163.00 ; \mathrm{n}=6)$ at 3 months. The mean percent change $(95 \% \mathrm{CI})$ in $\mathrm{P} 1 \mathrm{NP}$ level from baseline to 3 months in patients with stage $4 \mathrm{CKD}$ was $251.9 \%$ $(68.2 \%-435.7 \% ; n=6)$. In patients with stage $5 \mathrm{CKD}$, no data on median P1NP levels or the mean percent change in P1NP level were available. Among patients with severe stages of CKD for whom P1NP measurements were available at both baseline and 3 months $(n=6)$, individual levels increased in all but 1 patient (Figure 2).

\section{Bone mineral density}

Increases in BMD were also observed among patients with severe stages of $\mathrm{CKD}$, both overall and in individual patients. Mean (SD) BMD in patients with stage $4 \mathrm{CKD}$ increased 


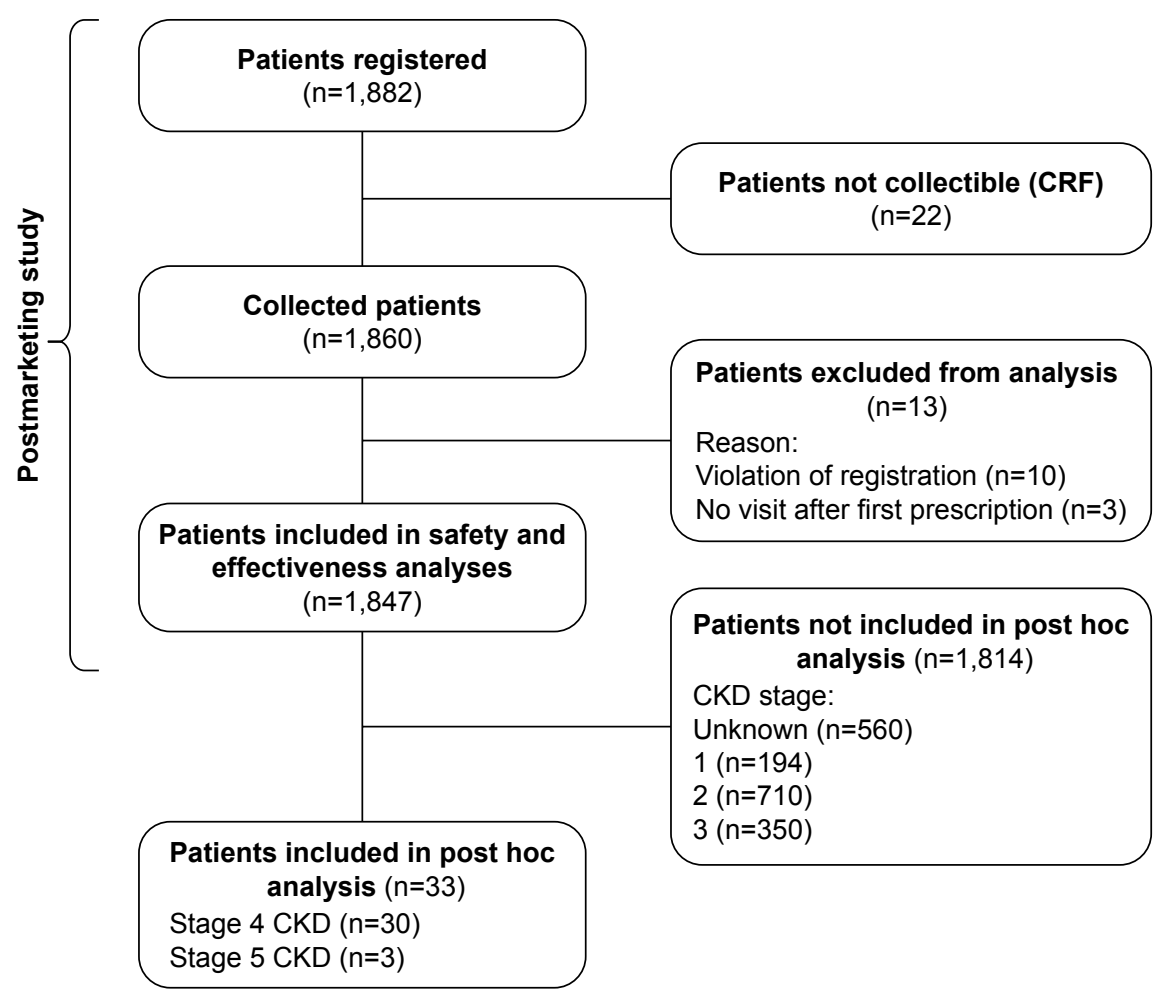

Figure I Patient disposition.

Abbreviations: CKD, chronic kidney disease; CRF, case report form.

from $0.805 \mathrm{~g} / \mathrm{cm}^{2}(0.208 ; \mathrm{n}=10)$ at baseline to $0.880 \mathrm{~g} / \mathrm{cm}^{2}$ $(0.144 ; n=9)$ at LOCF. In patients with stage $5 \mathrm{CKD}$, mean (SD) BMD increased from $0.968 \mathrm{~g} / \mathrm{cm}^{2}(\mathrm{n}=1)$ to $0.984 \mathrm{~g} / \mathrm{cm}^{2}$ $(n=3)$ at LOCF. The mean percent change (SD, 95\% CI) in BMD from baseline to LOCF was $22.2 \%$ (34.9, -14.4 to 58.8 ) in patients with stage 4 CKD and $1.6 \%$ in 1 patient with stage $5 \mathrm{CKD}$. Among patients with stage $4 \mathrm{CKD}$ for whom BMD measurements were available at both baseline and LOCF $(n=6)$, individual values increased in all but 1 patient (Figure 3 ).

\section{Fracture incidence}

Over the whole observation period of the PMS study, no fractures were observed in patients with stage $4 \mathrm{CKD}$ and 1 nonvertebral fracture of the ischium was observed in a patient with stage $5 \mathrm{CKD}$.

\section{Discussion}

This is the first report to focus on safety and effectiveness profiles of teriparatide treatment in Japanese patients with osteoporosis and severe stages of CKD. Regarding safety, the incidence of ADRs in patients with severe stages of CKD was $12.1 \%$ (4/33) and the incidence of ADRs in patients from the overall PMS study population was $7.58 \%(140 / 1,847)$.
Further, the type of ADRs (eg, hyperuricemia and headache) that were noted in patients with severe stages of CKD in this analysis were consistent with the known safety profile of teriparatide. ${ }^{21}$ In a subanalysis of the Fracture Prevention Trial (FPT), which enrolled 736 postmenopausal women with osteoporosis, the incidences of both treatment-emergent and renal-related adverse events were generally similar among patients with nil, mild, and moderate renal impairment, apart from higher incidences of hyperuricemia and hypercalcemia in patients with moderate renal impairment. ${ }^{22}$ Overall, the safety results of the current post hoc analysis concur with those of the FPT analysis. Specifically, no additional safety concerns were observed in this post hoc analysis in patients with severe stages of CKD, although careful administration of teriparatide is required when treating patients with osteoporosis and CKD.

Regarding the effectiveness of teriparatide, P1NP, a marker of bone formation, increased overall and in nearly all patients with evaluable measurements in this post hoc analysis. The increase in P1NP was observed in the first 3 months of treatment, which has been shown to be a sensitive and an accurate predictor of BMD response at 18 months. ${ }^{26}$ Similarly, BMD, the key assessment outcome of osteoporosis treatment, also increased both overall and in most patients. 
Table I Baseline demographics and disease characteristics of patients with osteoporosis and stage 4 or 5 CKD at high risk of fracture in Japan

\begin{tabular}{|c|c|c|}
\hline Characteristic & CKD stage $4\left(n=30^{a}\right)$ & CKD stage $5\left(n=3^{a}\right)$ \\
\hline \multicolumn{3}{|l|}{ Age } \\
\hline Mean (SD), years & $79.8(6.7)$ & $83.0(11.1)$ \\
\hline Median (min, max), years & $78.5(67,95)$ & $81(73,95)$ \\
\hline$<65$ years, $\mathrm{n}(\%)$ & $0(0.0)$ & $0(0.0)$ \\
\hline 65 to $<75$ years, $n(\%)$ & $7(23.3)$ & I (33.3) \\
\hline 75 to $<85$ years, $\mathrm{n}(\%)$ & $15(50.0)$ & I (33.3) \\
\hline$\geq 85$ years, $\mathrm{n}(\%)$ & $8(26.7)$ & I (33.3) \\
\hline \multicolumn{3}{|l|}{ Height } \\
\hline Mean (SD), cm & $148.2(5.1)\left(n=16^{b}\right)$ & I49.7 (5.9) $\left(n=3^{b}\right)$ \\
\hline \multicolumn{3}{|l|}{ Weight } \\
\hline Mean (SD), kg & $49.2(13.0)\left(n=17^{b}\right)$ & $45.0(5.6)\left(n=3^{b}\right)$ \\
\hline Female, $\mathrm{n}(\%)$ & $30(100.0)$ & $3(100.0)$ \\
\hline \multicolumn{3}{|l|}{ Menopause status, n (\%) } \\
\hline Premenopause & $0(0.0)$ & $0(0.0)$ \\
\hline Postmenopause & $26(86.7)$ & $3(100.0)$ \\
\hline Unknown & $4(13.3)$ & $0(0.0)$ \\
\hline Age at menopause, mean (SD), years & $47.0(5.9)$ & $50.0^{c}$ \\
\hline History of fracture, n (\%) & $24(80.0)$ & $3(100.0)$ \\
\hline History of proximal femoral fracture in patients' parents, $n(\%)$ & I (3.3) & $0(0.0)$ \\
\hline \multicolumn{3}{|l|}{ Vertebral fractures, $\mathrm{n}(\%)$} \\
\hline I & $6(20.0)$ & I (33.3) \\
\hline 2 & $5(16.7)$ & I (33.3) \\
\hline$\geq 3$ & $4(13.3)$ & I (33.3) \\
\hline \multicolumn{3}{|l|}{ Nonvertebral fractures, n (\%) } \\
\hline I & $4(13.3)$ & $2(66.7)$ \\
\hline 2 & $2(6.7)$ & $0(0.0)$ \\
\hline$\geq 3$ & $0(0.0)$ & $0(0.0)$ \\
\hline \multicolumn{3}{|l|}{ Femoral neck BMD, \% of YAM, n (\%) } \\
\hline$\geq 80 \%$ & $5(16.7)$ & $2(33.3)$ \\
\hline$\geq 70 \%$ to $<80 \%$ & $4(13.3)$ & $0(0.0)$ \\
\hline$<70 \%$ & $8(26.7)$ & $0(0.0)$ \\
\hline Current smoker, n (\%) & $0(0.0)$ & $0(0.0)$ \\
\hline Alcohol consumption (>3 U/day), n (\%) & $0(0.0)$ & $0(0.0)$ \\
\hline \multicolumn{3}{|l|}{ Creatinine } \\
\hline Mean (SD), $\mu \mathrm{mol} / \mathrm{L}$ & $1.58(0.22)(n=30)$ & $5.37(3.21)(n=3)$ \\
\hline Median (min, max), $\mu \mathrm{mol} / \mathrm{L}$ & I.5। $(1.30,2.29)$ & $4.60(2.6 I, 8.90)$ \\
\hline \multicolumn{3}{|l|}{ ALP } \\
\hline Mean (SD) & $306.7(n=26)$ & $604(n=2)$ \\
\hline Median (min, $\max$ ) & $261.0(101,835)(n=26)$ & $604.0(554,654)(n=2)$ \\
\hline
\end{tabular}

Notes: annless otherwise specified. 'Patients whose height and weight were measured earlier than 4 weeks before teriparatide administration were not included. 'Data based on I patient only.

Abbreviations: ALP, alkaline phosphatase; BMD, bone mineral density; CKD, chronic kidney disease; max, maximum; min, minimum; $\mathrm{n}$, number; SD, standard deviation; YAM, young adult mean.

Nonvertebral fracture was observed in 1 out of 33 patients during the observation period in this study. The FPT subanalysis also found that teriparatide had similar effects on P1NP, $\mathrm{BMD}$, and fracture incidence in patients with and without renal impairment. ${ }^{22}$ The results of this post hoc analysis and those of the FPT analysis are therefore also in general agreement with respect to effectiveness measures.

Elderly patients with osteoporosis also commonly have concomitant medical problems, including CKD, which has also been shown to increase in incidence with age and is independently associated with increased fracture risk. ${ }^{9}, 12,13$ Therefore, in patients at risk of fracture, treatment is required for osteoporosis and for $\mathrm{CKD}$, because this condition also reduces bone density and bone quality. In practice, however, treatment decisions for patients with osteoporosis and severe stages of CKD are difficult for several reasons. First, treatment options for preventing secondary fractures among patients with severe stages of CKD are limited in the real-world 


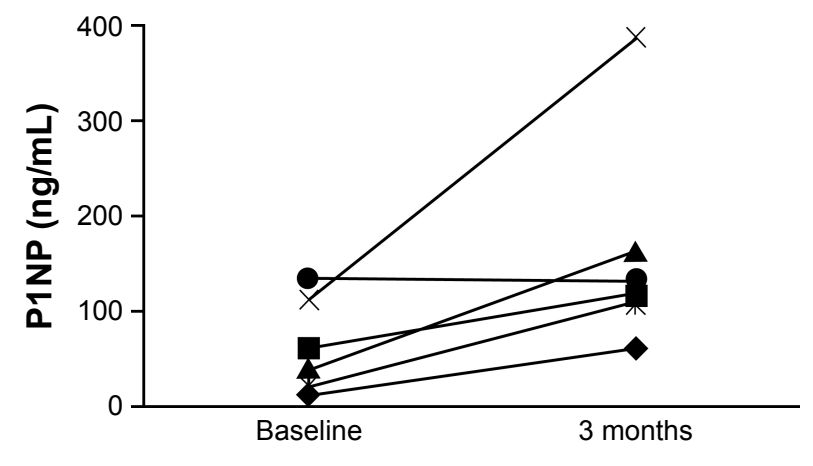

Figure 2 Change in PINP level from baseline to 3 months for individual patients with evaluable measurements.

Abbreviation: PINP, procollagen type I N-terminal propeptide.

clinical setting. In particular, according to the prescribing information, some bisphosphonates are contraindicated, whereas others are not recommended in patients with severe stages of CKD. ${ }^{14,27}$ Second, it has been estimated that $\sim 1$ in 3 patients with osteoporosis are not treated with osteoporosis medication. ${ }^{28}$ Likewise, despite the $59 \%$ estimated lifetime risk of having a GFR $<60 \mathrm{~mL} / \mathrm{min}$ per $1.73 \mathrm{~m}^{2}$, CKD is not always accurately assessed in clinical practice because GFR is not required to calculate World Health Organization Fracture Risk Assessment Tool (WHO FRAX ${ }^{\mathrm{TM}}$ ) scores. ${ }^{29}$ Consequently, diagnosis and treatment of osteoporosis are challenging in patients with severe stages of CKD because of limited evidence and the possibility of renal bone disease obscuring the clinical picture. ${ }^{30}$ For patients with osteoporosis and severe stages of CKD, teriparatide is a possible treatment option, but there is little published evidence focusing on the effectiveness and safety profiles of teriparatide. In addition to investigating the actual use of teriparatide in a real-world clinical setting in Japan, we sought to investigate

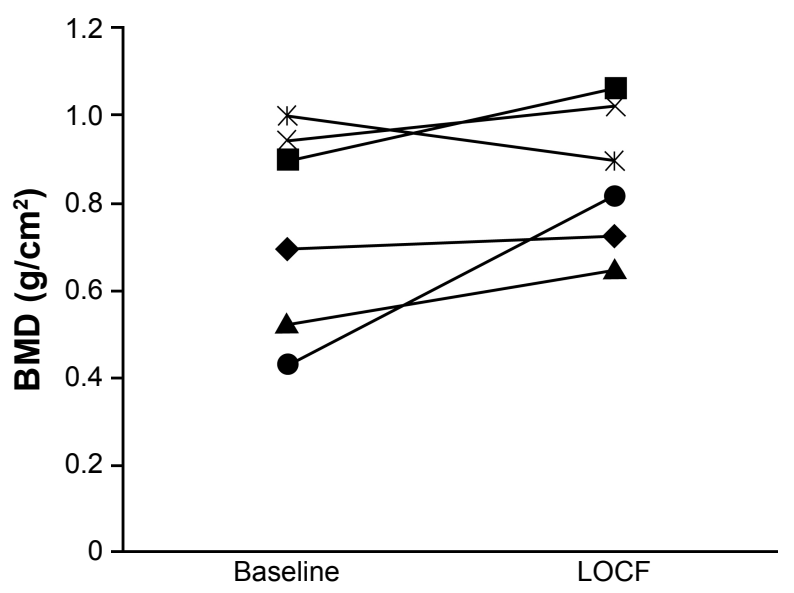

Figure 3 Change in least squares mean BMD from baseline to LOCF for individual patients with evaluable measurements.

Abbreviations: BMD, bone mineral density; LOCF, last observation carried forward. the effectiveness and safety of teriparatide in patients with osteoporosis and comorbid severe stages of CKD. In this post hoc analysis, teriparatide was associated with a positive response in terms of effectiveness and no additional safety concerns were observed.

Several limitations of this post hoc analysis should be pointed out. First, these results lack statistical power because of the small number of enrolled patients, particularly those with stage 5 CKD. Further, information was not always available for individual patients at each evaluation point because data were collected from investigator reports. The methods of evaluating serum P1NP, BMD, and fractures may also have varied between investigator sites. In addition to these specific limitations, post hoc analyses and observational studies also have several general limitations. Importantly, a lack of randomization inherent in these types of studies makes proper statistical inference of causality of apparent treatment effects difficult. These types of studies are also susceptible to reporting bias, and the lack of a comparator group makes it further difficult to assign a causal relationship to possible treatment effects. Notwithstanding these limitations, our findings are expected to help physicians and surgeons evaluate the advantages and potential disadvantages of prescribing teriparatide for osteoporosis in Japanese patients with severe stages of CKD.

\section{Conclusion}

This post hoc analysis found that teriparatide showed positive effects on key outcomes and markers of osteoporosis in Japanese patients with osteoporosis and severe stages of CKD. Further, no additional safety concerns were observed with daily teriparatide in this study. However, physicians and surgeons should continue to consider the balance between advantages and possible disadvantages when prescribing teriparatide to patients with renal impairment or hypercalcemia.

\section{Acknowledgments}

This study was sponsored by Eli Lilly Japan K.K., manufacturer/licensee of teriparatide $\left(\right.$ Forteo $\left.^{\circledR}\right)$. Medical writing assistance was provided by Mark Snape, MB BS, CMPP, and Rebecca Lew, PhD, CMPP, of ProScribe Envision Pharma Group, and was funded by Eli Lilly Japan K.K. ProScribe's services complied with international guidelines for Good Publication Practice (GPP3). Eli Lilly Japan K.K. was involved in the study design, data collection, data analysis, and preparation of the manuscript. The authors would like to thank all study patients and investigators who participated in the study. 


\section{Author contributions}

All the authors were involved in the study design and contributed to drafting of the manuscript. HE, FY, and KK were involved in the interpretation of study data, and MT conducted the statistical analysis. All authors contributed toward data analysis, drafting and critically revising the paper and agree to be accountable for all aspects of the work.

\section{Disclosure}

All authors are employees of Eli Lilly Japan K.K. The authors report no other conflicts of interest in this work.

\section{References}

1. National Institutes of Health. Osteoporosis prevention, diagnosis, and therapy. NIH Consens Statement (online). 2000;17(1):1-36.

2. Fujiwara S. Epidemiology of osteoporosis in Japan. J Bone Miner Metab. 2005;23(suppl):81-83.

3. Orimo H, Nakamura T, Hosoi T, et al. Japanese 2011 guidelines for prevention and treatment of osteoporosis - executive summary. Arch Osteoporos. 2012;7(1-2):3-20.

4. Nojiri S, Burge RT, Flynn JA, Foster SA, Sowa H. Osteoporosis and treatments in Japan: management for preventing subsequent fractures. J Bone Miner Metab. 2013;31(4):367-380.

5. Johnell O, Kanis JA. An estimate of the worldwide prevalence and disability associated with osteoporotic fractures. Osteoporos Int. 2006; 17(12):1726-1733.

6. Imai E, Horio M, Watanabe T, et al. Prevalence of chronic kidney disease in the Japanese general population. Clin Exp Nephrol. 2009;13(6): 621-630.

7. Jones CA, McQuillan GM, Kusek JW, et al. Serum creatinine levels in the US population: third National Health and Nutrition Examination Survey. Am J Kidney Dis. 1998;32(6):992-999.

8. Nishikawa A, Ishida T, Taketsuna M, Yoshiki F, Enomoto H. Safety and effectiveness of daily teriparatide in a prospective observational study in patients with osteoporosis at high risk of fracture in Japan: final report. Clin Interv Aging. 2016;11:913-925.

9. Dukas L, Schacht E, Stahelin HB. In elderly men and women treated for osteoporosis a low creatinine clearance of $<65 \mathrm{ml} / \mathrm{min}$ is a risk factor for falls and fractures. Osteoporos Int. 2005;16(12):1683-1690.

10. Looker AC, Orwoll ES, Johnston CC Jr, et al. Prevalence of low femoral bone density in older U.S. adults from NHANES III. J Bone Miner Res. 1997;12(11):1761-1768.

11. Nickolas TL, McMahon DJ, Shane E. Relationship between moderate to severe kidney disease and hip fracture in the United States. J Am Soc Nephrol. 2006;17(11):3223-3232.

12. Ensrud KE, Lui LY, Taylor BC, et al; Osteoporotic Fractures Research Group. Renal function and risk of hip and vertebral fractures in older women. Arch Intern Med. 2007;167(2):133-139.

13. Fried LF, Biggs ML, Shlipak MG, et al. Association of kidney function with incident hip fracture in older adults. J Am Soc Nephrol. 2007;18(1): 282-286.
14. Orimo H. Japanese 2015 Guidelines for Prevention and Treatment of Osteoporosis. Japan: Life Science Publishing Co., Ltd; 2015.

15. Miller PD. Bone disease in CKD: a focus on osteoporosis diagnosis and management. Am J Kidney Dis. 2014;64(2):290-304.

16. Bodenner D, Redman C, Riggs A. Teriparatide in the management of osteoporosis. Clin Interv Aging. 2007;2(4):499-507.

17. Hodsman AB, Bauer DC, Dempster DW, et al. Parathyroid hormone and teriparatide for the treatment of osteoporosis: a review of the evidence and suggested guidelines for its use. Endocr Rev. 2005;26(5): 688-703.

18. Krege JH, Wan X. Teriparatide and the risk of nonvertebral fractures in women with postmenopausal osteoporosis. Bone. 2012;50(1): $161-164$.

19. Miyauchi A, Matsumoto T, Sugimoto T, Tsujimoto M, Warner MR, Nakamura T. Effects of teriparatide on bone mineral density and bone turnover markers in Japanese subjects with osteoporosis at high risk of fracture in a 24-month clinical study: 12-month, randomized, placebo-controlled, double-blind and 12-month open-label phases. Bone. 2010;47(3):493-502.

20. Walsh JB, Lems WF, Karras D, et al. Effectiveness of teriparatide in women over 75 years of age with severe osteoporosis: 36-month results from the European Forsteo Observational Study (EFOS). Calcif Tissue Int. 2012;90(5):373-383.

21. Forteo ${ }^{\circledR}$ [prescribing information]. Kobe, Japan: Eli Lilly Japan K.K.; 2014. Available from: https://www.lilly.co.jp/_Assets/pdf/ lillyanswers/products/tenpu_for-kit600.pdf. Accessed September 30, 2016. Japanese.

22. Miller PD, Schwartz EN, Chen P, Misurski DA, Krege JH. Teriparatide in postmenopausal women with osteoporosis and mild or moderate renal impairment. Osteoporos Int. 2007;18(1):59-68.

23. Orimo H, Hayashi Y, Fukunaga M, et al. Diagnostic criteria for primary osteoporosis: year 2000 revision. J Bone Miner Metab. 2001;19(6): 331-337.

24. National Kidney Foundation. K/DOQI Clinical practice guidelines for chronic kidney disease: evaluation, classification, and stratification. Am J Kidney Dis. 2002;39:S1-S266.

25. Matsuo S, Imai E, Horio M, et al; Collaborators developing the Japanese equation for estimated GFR. Revised equations for estimated GFR from serum creatinine in Japan. Am J Kidney Dis. 2009;53(6):982-992.

26. Chen P, Satterwhite JH, Licata AA, et al. Early changes in biochemical markers of bone formation predict BMD response to teriparatide in postmenopausal women with osteoporosis. J Bone Miner Res. 2005; 20(6):962-970.

27. Ott SM. Therapy for patients with CKD and low bone mineral density. Nat Rev Nephrol. 2013;9(11):681-692.

28. Sato M, Vietri J, Flynn JA, Fujiwara S. Treatment for osteoporosis among women in Japan: associations with patient characteristics and patient-reported outcomes in the 2008-2011 Japan National Health and Wellness Surveys. J Osteoporos. 2014;2014:909153.

29. Grams ME, Chow EK, Segev DL, Coresh J. Lifetime incidence of CKD stages 3-5 in the United States. Am J Kidney Dis. 2013;62(2): $245-252$.

30. Modi A, Sajjan S, Gandhi S. Challenges in implementing and maintaining osteoporosis therapy. Int J Womens Health. 2014;6:759-769.
Clinical Interventions in Aging

\section{Publish your work in this journal}

Clinical Interventions in Aging is an international, peer-reviewed journal focusing on evidence-based reports on the value or lack thereof of treatments intended to prevent or delay the onset of maladaptive correlates of aging in human beings. This journal is indexed on PubMed Central, MedLine,

\section{Dovepress}

CAS, Scopus and the Elsevier Bibliographic databases. The manuscript management system is completely online and includes a very quick and fair peer-review system, which is all easy to use. Visit http://www.dovepress. com/testimonials.php to read real quotes from published authors. 